\title{
A Method for Randomly Hiding Secret Encrypted Data in Images using Cellular Automata
}

\author{
Ansam O. Abdulmajeed \\ Fatima M. Abdullatif \\ Wafaa A. Mustafa \\ ansam_osamah@uomosul.edu.iq fatimamaan4@gmail.com \\ wfwf9255@gmail.com \\ Department of Software Engineering \\ College of Computer Sciences and Mathematics \\ University of Mosul, Mosul, Iraq
}

Received on: 13/06/2018

Accepted on: 24/01/2019

\begin{abstract}
The present research was aimed to design and implement an algorithm that combines cryptography and steganography to achieve a higher level of security. The algorithm hid encrypted text into color images in a scattered manner based on randomly generated numbers. The rules of cellular automata were used to encrypt the secret text and generate random numbers. Each character of a secret text was treated as a cellular automaton and the rule 153 was used to encrypt it with the help of a secret key. The encrypted text was hide in the red plane of the cove image after generating random numbers by applying rules 30, 60, 90 as a hybrid cellular automata using the same secret key as an initial seed. The results were tested on a number of images that shown that the algorithm hide the secret text in the images without distorting it clearly, the algorithm also retrieved the entire secret text without any loss. It was concluded that the use of hybrid cellular automata is better in generating random numbers than using uniform cellular automata. It was also concluded that applying the retrieval algorithm with any minor changes in the value of the secret key affects the output of the decryption and the output of random number generation and causes a significant difference in the retrieved text.
\end{abstract}

Keyword: cryptography, steganography, cellular automata.

$$
\begin{aligned}
& \text { طريقة لإخفاء البيانات السرية المشفرة عشوائيا في الصور باستخدام الآلات الخلوية } \\
& \text { وفاء عبد اللطيف مصطفى } \\
& \text { فاطمة معن عبد اللطيف } \\
& \text { أنسام أسامة عبد المجيد } \\
& \text { قسم هندسة البرمجيات } \\
& \text { كلية علوم الحاسوب والرياضيات } \\
& \text { جامعة الموصل، الموصل، العراق }
\end{aligned}
$$

\section{الملخص}


وباستخدام المفتاح السري نفسه كبذرة أولية. اختُرت النتائج على صورٍ عدة. وتبين أن الخوارزمية أخفت النص

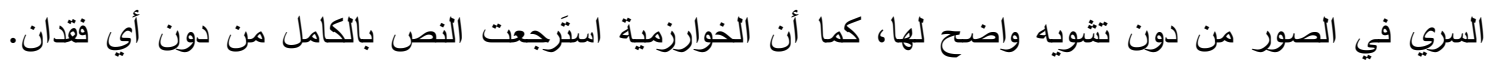

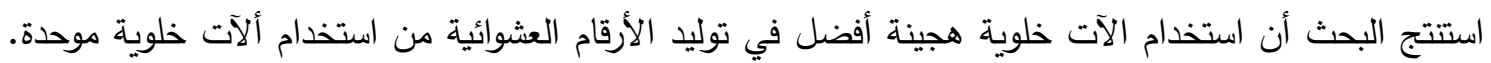

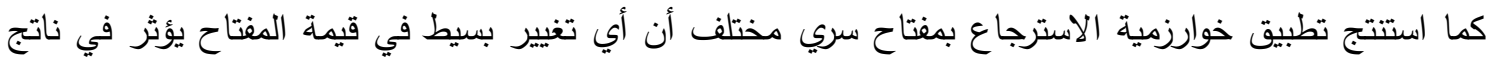

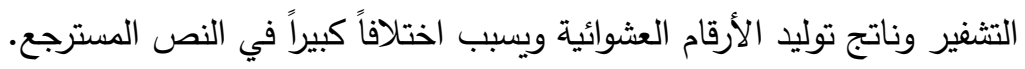
الكلمات المفتاحية: التثفير ، إخفاء المعلومات، الآلات الخلوية.

\section{Introduction 1 المقدمة}

أصبح الانترنيت، في الوقت الحاضر، جزءاً لا يتجزأ من حياة الكثيرين، ومطلباً أساسياً في العديد من قطاعات الأعمال كالأعمال المصرفية والتسوق. وعلى الرغم من مزايا استخدام الانترنت العديدة، إلا أن له ألهاء أثراً

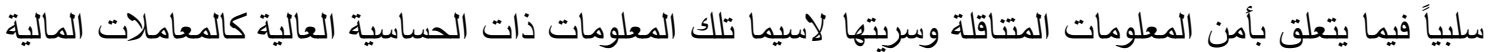

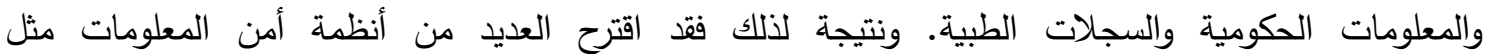

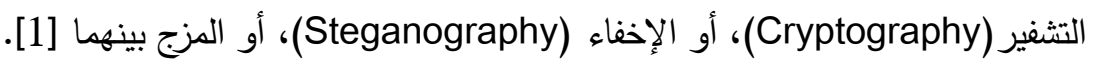

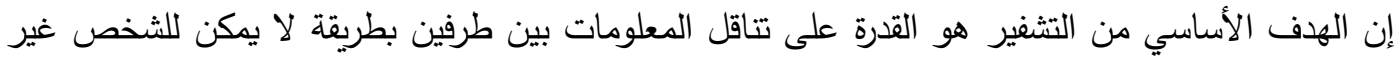

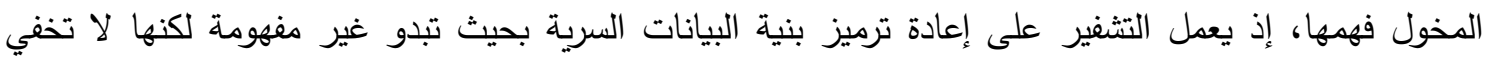

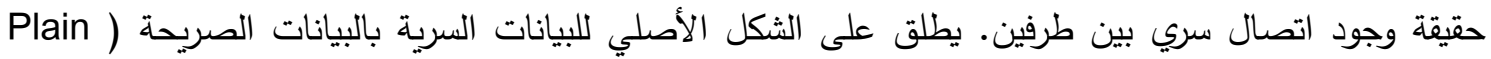

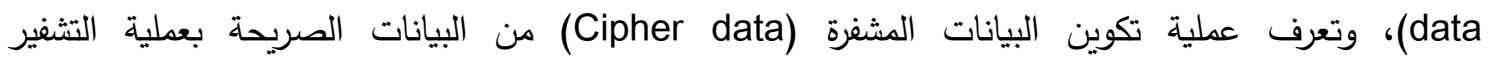

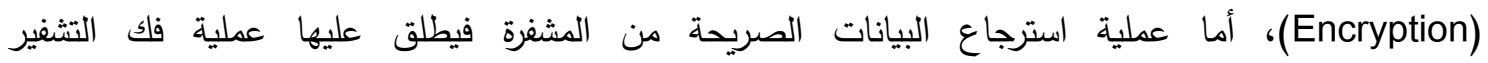

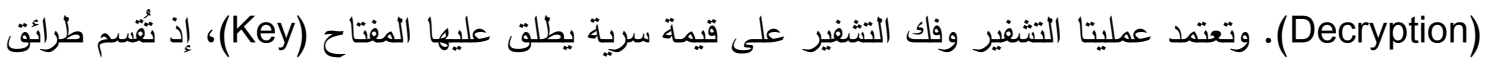
التثفير بحسب الففتاح إلى قسمين: التشفير المتماثل (Symmetric Cryptography) الذي يستخدم الفنتاح نفسه في عمليتي التثفير وفك التثفير، والتثفير غير الكتماثل (Asymmetric Cryptography) الذي يستخدم مفتاحين مختلفين لكل عملية [2]. أما الإخفاء فهو تقانة استخدام وسطِ ما يدعى الغطاء (Cover) لإخفاء بيانات سرية داخله بصورة لا يمكن

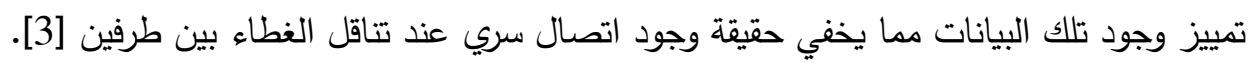
يهدف البحث إلى تطبيق خوارزمية تدمج بين التثفير والإخفاء لتحقيق مستوى أعلى من الأمنية، إذ تستخدم

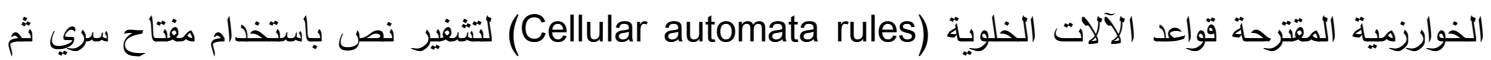
إخفائه بطريقة مبثثرة في صور ملونة، باعتبارها غطاء للبيانات السرية، اعتماداً على أرقام مولدة عشوائياً باستخدام قواعد الآلات الخلوية أيضاً.

ورقة البحث مقسمة على النحو الآتي: الفقرة الثانية تتناول دراسات سابقة ذات علاقة بموضوع البحث، أما الفقرة الثالثة فتثتمل على تعريف بالآلات الخلوية وأهم خصائصها. وتسرد الفقرة الرابعة الخوارزمية المقترحة. أما النتائج ومناقتتها فتأتي ضمن الفقرة الخامسة. وتتناول الفقرة الأخيرة أهم الاستنتاجات التي تم التوات التوصل إليها. 
2 2. - 20 - دراسات سابقة

قدَّم .Shanmugasundaram et al خوارزمية تتضمن تحويل البيانات النصية إلى تسلسل الحمض النووي

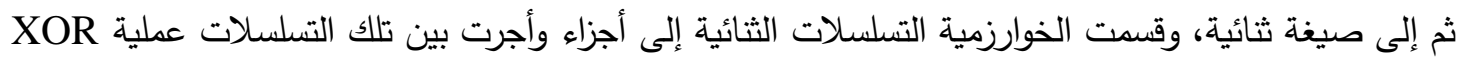

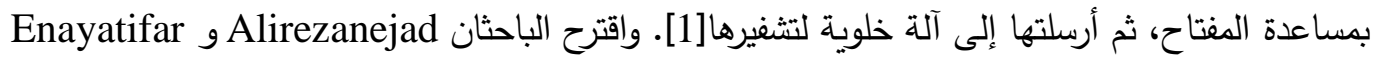
خوارزمية دمجت بين الآلات الخلوية وبين دوال الفوضى لتحديد الموضع المناسب من الصورة لاستخدامه في

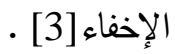

وقام Bouchkaren و Lazaar بتقسيم النص غير المشفر إلى مجموعة من الكتل وتحويلها إلى مصفوفات

ثنائية ومن ثم تشفيرها باستخدام الآلات الخلوية ثنائية البعد[4].

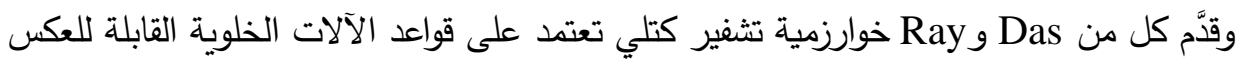

(reversible cellular automata rules) عملية فك التشفير بقواعد معاكسة للقواعد المستخدمة في التشفير ؛ إذ إن عكس قاعدة 240 هي 15 وعكس قاعدة 204 هي 51، وعكس قاعدة 170 هي 58 [5]. أما Amirthalingama و وفد قدما خوارزمية تثفير تتضمن تحويل النص إلى كتل تتكون من مجموعة حروف ثم تحويل الحروف في كل كتلة إلى صيغة ثنائية ثم تقسيمها إلى مستويات وتثفير كل مستوى باستخدام الآلات الخلوية [6].

البحث الحالي عامَل كل حرف من النص السري معاملة آلة خلوية وشفره باستخدام مفتاح سري يتكون من

24-bits قُمِّم إلى ثلاثة أقسام بطول 8-bits كي تتتاسب مع الحرف السري وقد أخفي النص المشفر بطريقة مبعثرة بحسب أرقام مولدة عشوائياً على أساس قيمة المفتاح السري نفسه وباستخدام ثلاث قواعد مختلفة.

\section{Cellular Automata الآلات الخلوية}

تستخدم الآلات الخلوية (Cellular automata) على نطاق واسع في العديد من المجالات مثل إنثاء الرسومات ، والتعرف على الأنماط، والتشفير، إذ يمكن الاستفادة من مميزات الآلات الخلوية في توليد أرقام

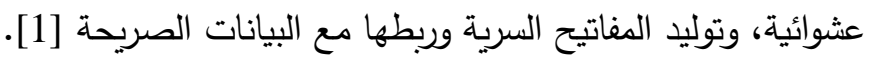
الآلات الخلوية هي نظام ديناميكي مرتب بثكل شبكة مكونة من N من الخلايا (Cells) المتجاورة (انظر:

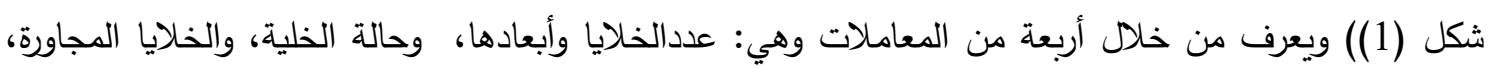

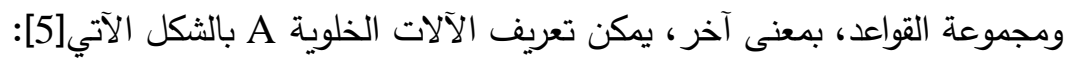
$A=\{S, Z, f, V\}$

S S : حالة الخلية، وهي الأبجدية المكونة للآلة الخلوية على سبيل المثال S Z f: دالة الحالة التالية (next state function) وتتمثل بقواعد الآلة الخلوية. والقواعد عبارة عن عمليات منطقية تتفذ على قيم الخلايا المتجاورة. V : مجموعة الخلايا المجاورة للخلية الحالية. 
تطبق قواعد الآلة الخلوية على الخلايا باستمرار لإنتاج جيل جديد من الخلايا، وتعتمد حالة الخلية الحالية

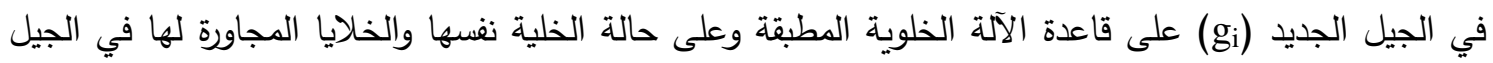
السابق (gi-1)

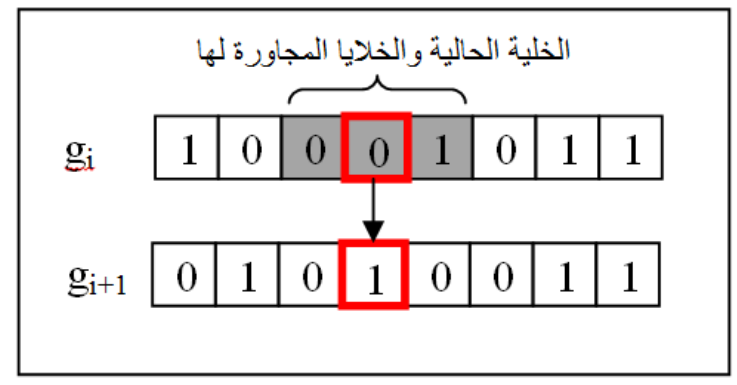

\section{الشكل (1): شكل يوضح جيلين لآلة خلوية أحادية البعد[7]}

تتأثر كل خلية في الآلة الخلوية أحادية البعد في كل جيل بالخلية نفسها من الجيل السابق وبالخلايا المجاورة

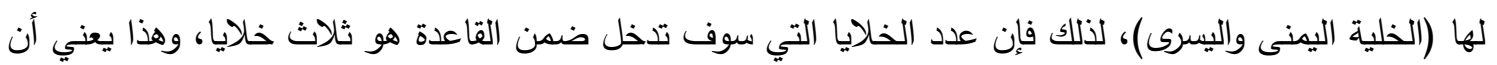
هناك ثمانية أنماط مختلفة تشكلها تلك الخلايا المتجاورة ومن ثم يمكن تطبيق 256 قاعدة مختلفة على آلى آلة خلوية أحادية البعد[5]. والثكل 2 يوضح تطبيق قاعدة رقم 126 على جميع الأنماط المختلفة للخلايا المتجاورة؛ إذ تمثل الخلية السوداء الرقم 1 وتمثل الخلية البيضاء الرقم 0.

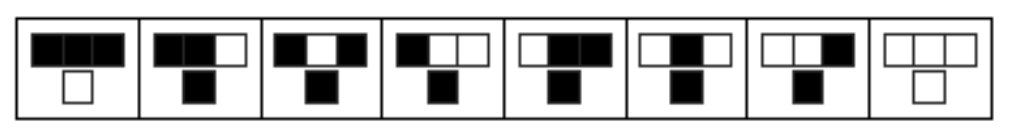

الثكل (2): شكل يوضح القاعدة رقم 126 [1].

من الثكل 2 نلاحظ أن الخلايا الناتجة تمثل الرقم12610 = 201111110 ومن هنا أتت تسمية القواعد. تصنف الآلات الخلوية بحسب كيفية التعامل مع الخلايا في الحافات إلى[6]: 1- الآلة الخلوية ذات الحدود الفارغة (Null boundary cellular automata): توصل فيها الخلايا في

$$
\text { الحافات بالقيمة } 0 .
$$

2- الآلة الخلوية ذات الحدود الدورية (Periodic boundary cellular automata): توصل فيها الخلايا

$$
\text { في الحافات مع بعضها البعض. }
$$

كما تصنف الآلات الخلوية بحسب كيفية تطبيق القواعد على الخلايا المتجاورة إلى[6]:

1- الآلة الخلوية الموحدة (Uniform cellular automata): تطبق فيها القاعدة نفسها على الخلايا كلها. 2- الآلة الخلوية الهجينة (Hybrid cellular automata): تطبق فيها قواعد مختلفة على كل مجموعة من 
3- الآلة الخلوية الخطية (Linear cellular automata): تعتمد القاعدة المطبقة فيها على عملية XOR

فقط.

4- الآلة الخلوية المتممة (Complement cellular automata): تعتمد القاعدة المطبقة فيها على عملية

فقط. XNOR

Proposed Algorithm الخوارزمية المقترحة

استخدمت الخوارزمية المقترحة قواعد مختلفة لكل من التشفير وتوليد أرقام عشوائية، حيث إذ شفّر كل حرف

من حروف النص السري باستخدام مفتاح سري يتكون من 24-bits. ولكي يتاسب المفتاح السري مع تثفير كل

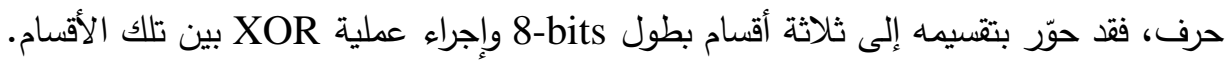

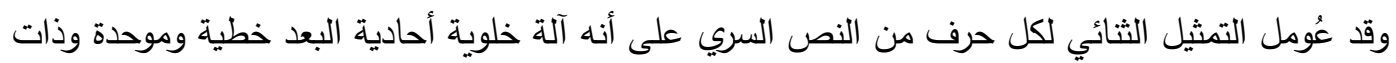

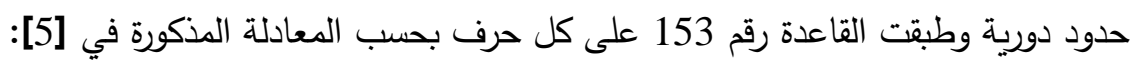
$\mathrm{q}_{\mathrm{i}}(\mathrm{g}+1)=\operatorname{NOT}\left(\mathrm{q}_{\mathrm{i}+1}(\mathrm{~g})\right.$ XOR $\left.\mathrm{q}_{\mathrm{i}}(\mathrm{g})\right)$

qi

g: (الجيل الحالي.

طبقت القاعدة 153 لعدد من المرات متفق عليه بين الطرفين، ثم أجريت عملية XOR للناتج مع قيمة الففتاح السري. وبعد إتمام عملية التشفير ، أخفي النص المشفر في صور ملونة بطريقة البت الأقل أهمية (LSB)

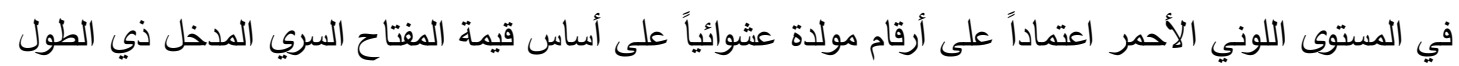
(24_bits) باعتباره البذرة (initial seed) وباستخدام ثلاث قواعد مختلفة وهي: قاعدة رقم 30، وقاعدة رقم 60، وقاعدة رقم 90 وبحسب المعادلات المذكورة في [5]:

$$
\begin{aligned}
\mathrm{q}_{i}(\mathrm{~g}+1)=\mathrm{q}_{\mathrm{i}-1}(\mathrm{~g}) \text { XOR }\left(\mathrm{q}_{i}(\mathrm{~g}) \text { OR } \mathrm{q}_{\mathrm{i}+1}(\mathrm{~g})\right) \ldots \ldots \ldots \ldots(2) & \text { Rule } 30 \\
\mathrm{q}_{\mathrm{i}}(\mathrm{g}+1)=\mathrm{q}_{\mathrm{i}-1}(\mathrm{~g}) \text { XOR } \mathrm{q}_{\mathrm{i}}(\mathrm{g}) \ldots \ldots \ldots \ldots \ldots \ldots \ldots \ldots(3) & \text { Rule } 60 \\
\left.\mathrm{q}_{\mathrm{i}}(\mathrm{g}+1)=\left(\mathrm{q}_{\mathrm{i}-1}(\mathrm{~g}) \text { XOR } \mathrm{q}_{\mathrm{i}}(\mathrm{g})\right) \text { XOR } \mathrm{q}_{i+1}(\mathrm{~g})\right) \ldots \ldots \ldots(4) & \text { Rule } 90
\end{aligned}
$$

إذ طُبقت القواعد الثلاثة آنفة الذكر على البذرة، باعتبارها آلة خلوية خطية هجينة وذات حدود دورية، بصورة

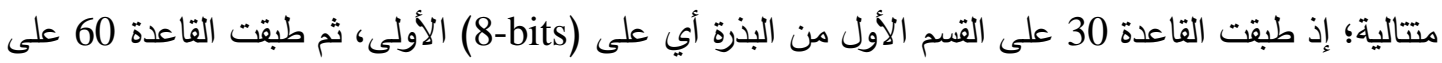

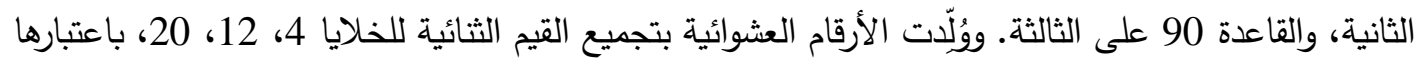
الخلايا الوسطية، بعد كل جيل (انظر الثكل 3).

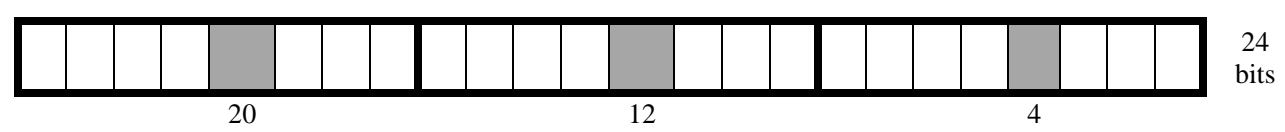

الثكل (3): شكل يوضح كيفية توليد الارقام العشوائية من المفتاح السري 
يقوم المستلم عن طريق المفتاح السري المتقق عليه باستخراج النص المشفر من الصورة المرسلة إليه بعد أن

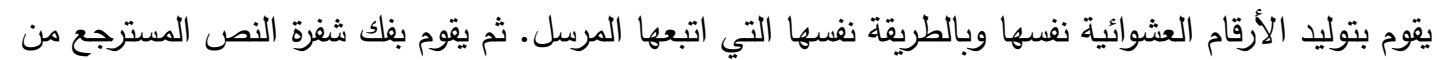
الصورة وبالمفتاح السري وبالطريقة نفسها التي استخدمها المرسل. والثكل (4) يوضح المخطط الانسيابي للخوارزمية المقترحة.

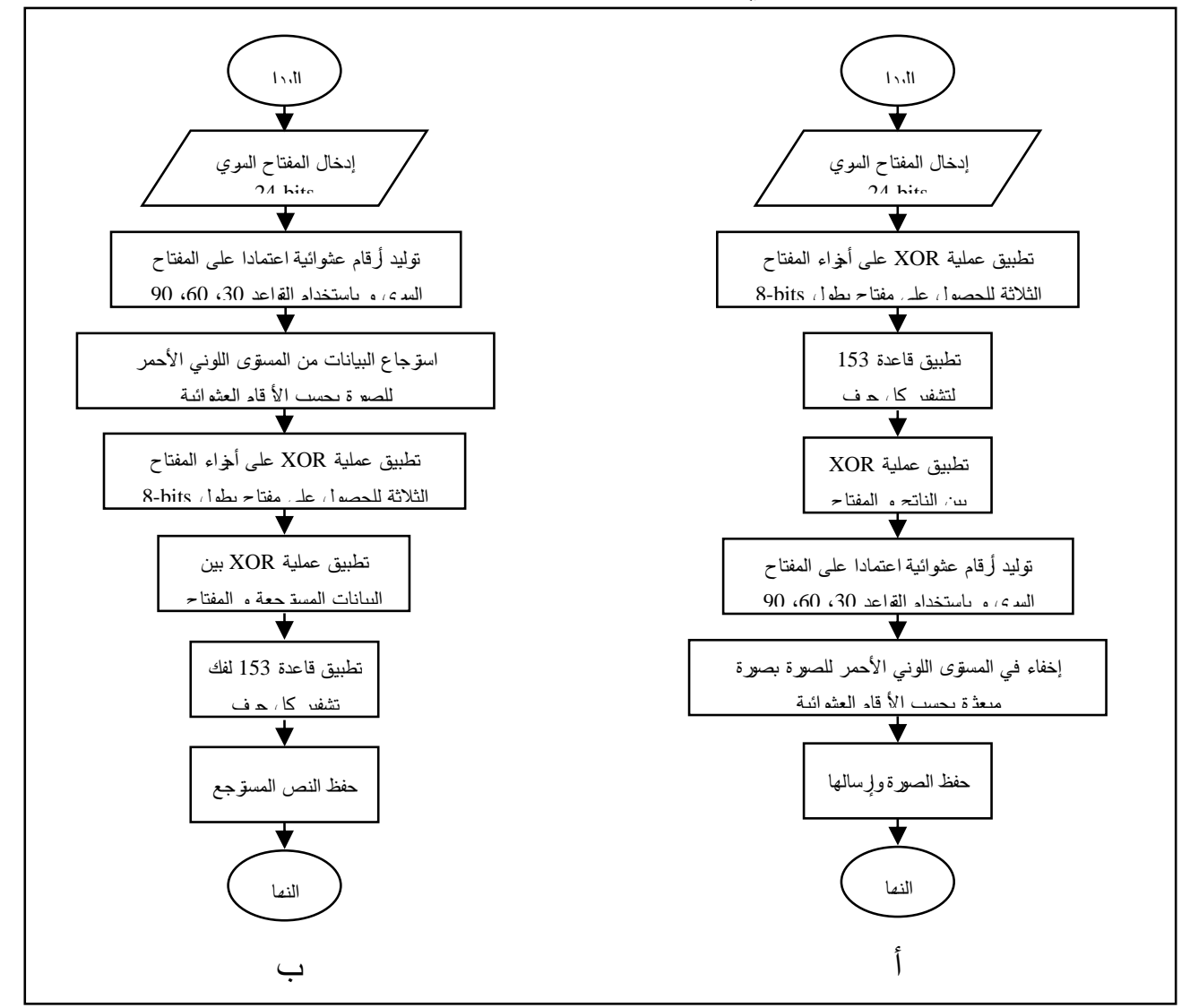

الثكل (4): المخطط الانسيابي للخوارزمية المقترحة

Results and discussion 5. النتائج والمناقشة

4672 bits أجريت اختبارات عدة للخوارزمية المقترحة على صور ملونة بالامتداد BMP لإخفاء نص بطول باستخدام قيم مختلفة من المفتاح السري، وكانت النتائج كما مبين في الجدول بالهدات (1). الجدول (1): نتائج تطبيق الخوارزمية المقترحة

\begin{tabular}{rrrrr}
\hline BER مقياس & PSNR مقياس & الصورة & Airplane \\
\hline 0 & 67.6578 & 9437119 & $512 \times 512$ & \\
0 & 62.6463 & 15478234 & $256 \times 256$ & Children
\end{tabular}




$0 \quad 65.1187 \quad 14789632 \quad 350 \times 350 \quad$ Strawberry

إن عملية الإخفاء لم تؤثر على جودة الصورة، وهذا يتبين من قيم مقياس PSNR الموضحة في الجدول (1)، كما

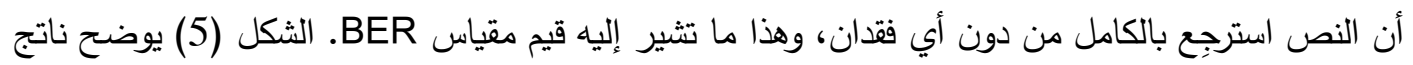
تطبيق الخوارزمية المقترحة على إحدى صور الاختبار • لن دون

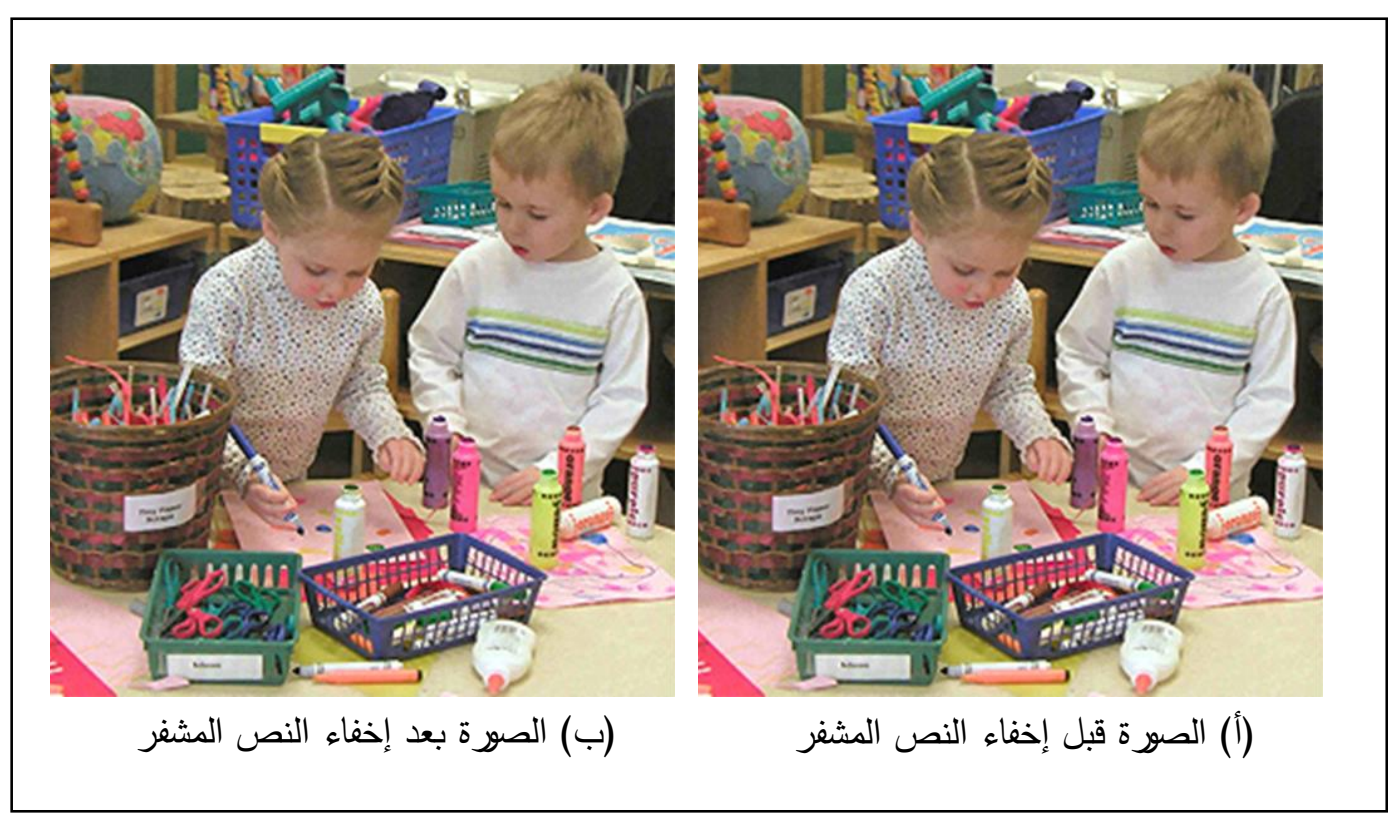

الشكل (5): صورة من صور الاختبارات قبل إخفاء النص المشفر وبعده

عمدت الخوارزمية المقترحة إلى استخدام آلة خلوية خطية هجينة مكونة من القواعد 30، و60، و 90 في توليد

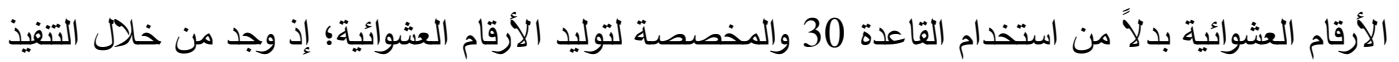

أن القاعدة 30 قد تصل إلى حالة مستقرة (steady state) بعد عدد معين من الأجيال وأن الدمج بين القواعد يعطي عددا أكبر من الأرقام العشوائية نتيجة لاستخدام عمليات منطقية مختلفة. والجدول (2) يوضح

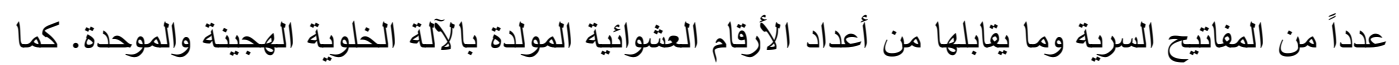

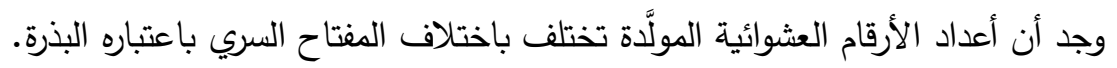
الجدول (2) أعداد الأرقام العشوائية باستخدام ألآت خلوية هجينة وموحدة

طول الأرقام العشوائية المولدة

الآلة الخلوية الموحدة المفتاح السري الآلة الغلوية الهجينة 
تعد الخوارزمية المقترحة حساسة لقيم المفتاح السري؛ إذ طُبقت خوارزمية الاسترجاع باستخدام مفتاح سري مختلف

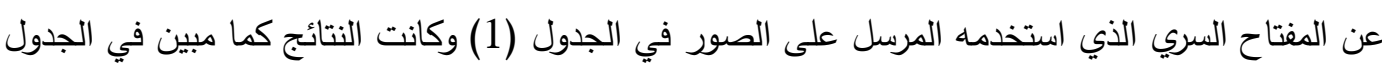

\begin{tabular}{rrr} 
الجدول (3): نتائج تطبيق خوارزمية الاسترجاع بمفتاح سري مختلف & \\
\hline BER Stego_Airplane \\
0.4899 & 9437219 & \\
0.4929 & 15475234 & Stego_Children \\
0.5098 & 14779632 & Stego_Strawberry \\
\hline
\end{tabular}

إن استخدام مفتاح سري عند الاسترجاع مختلف عن قيمته الأصلية المستخدمة في الإخفاء يؤدي إلى اختلاف واضح في النص المسترجع؛ لأن استخدام مفتاح سري مختلف يعني استخدام بذرة مختلفة في توليد الأرقام العشوائية ومن ثم خطأ في النص المسترجع إذ أن تلك الأرقام العشوائية تمثل المواقع التي تم الإخفاء فيها.

\section{Conclusions الاستنتاجات}

قدم البحث خوارزمية لإخفاء نص مشفر في صور ملونة بطريقة مبعثرة اعتماداً على أرقام مولدة عشوائياً. وقد

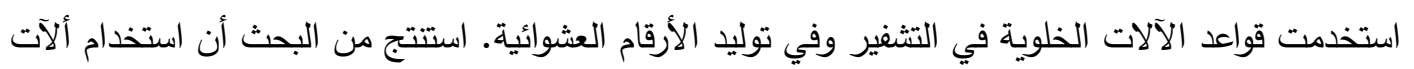

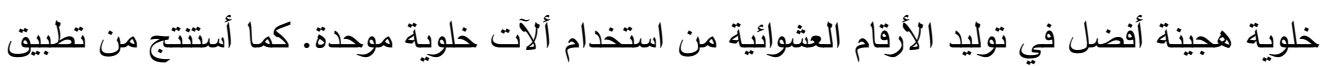
خوارزمية الاسترجاع بمفتاح سري مختلف أن أي تغيير بسيط في قيمة المفتاح يؤثر في ناتج التثفير وناتج

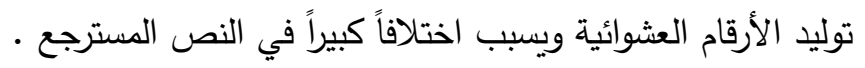




\section{المصادر}

[1] Shanmugasundaram G., Thiyagarajan P., and Pavithra S., (2015), "A Novel DNA Encryption System Using Cellular Automata ", International Journal of Security, Privacy and Trust Management (IJSPTM) Vol 4, No 3/4, pp.39-49.

[2] Mohini J., (2017), "Data Security Using 2D Cellular Automata Rules ", International Journal of Students' Research in Technology \& Management, Vol. 5, No. 1, pp: 5-8.

[3] M. Alirezanejad, and R. Enayatifar, (2012), "Steganography by using Logistic Map Function and Cellular Automata", Research Journal of Applied Sciences, Engineering and Technology, Vol. 4, No. 23, pp. 4991-4995.

[4] Bouchkaren S., and Lazaar S., (2014), " A Fast Cryptosystem Using Reversible Cellular

Automata ", International Journal of Advanced Computer Science and Applications,

Vol. 5, No. 5, pp.207-210.

[5] Das D., and Ray A., (2010), "A Parallel Encryption Algorithm for Block Ciphers Based on Reversible Programmable Cellular Automata", Journal of Computer Science and Engineering, Vol. 1, Issue 1, pp.82-90

[6] Amirthalingama S., Latha K., (2016), "A Study on Encryption Using ThreeDimensional Cellular Automata ", Journal. ScienceAsia, Vol. 42, pp.42-48

[7] Kar B., Rao D. C., and Rath A. K., (2011), "Generating PNS for Secret Key Cryptography Using Cellular Automaton", international Journal of Advanced Computer Science and Applications, Vol. 2, No. 5, pp.101-105. 